\title{
Elastic freedom in cellular solids and composite materials
}

Lakes, R. S., "Elastic freedom in cellular solids and composite materials", in Mathematics of Multiscale Materials, p. 129-153, ed. K. Golden, G. Grimmert, R. James, G. Milton, P. Sen, IMA vol. 99, Springer, NY, Berlin, (1998).

Roderic Lakes, Ph. D.

Wisconsin Distinguished Professor

Department of Engineering Physics

Engineering Mechanics Program; Biomedical Engineering Department

Materials Science Program and Rheology Research Center

University of Wisconsin-Madison

Engineering Research Building

1500 Engineering Drive, Madison, WI 53706-1687

web- http://silver.neep.wisc.edu/ lakes 


\begin{abstract}
The question of how much freedom is to be incorporated in a continuum theory must ultimately be decided by experiment. There are several theories which describe behavior of materials. An early uniconstant theory was proposed based on atomic interaction theory; it was abandoned since it predicted a Poisson's ratio of 1/4 for all materials. The elasticity theory currently accepted as classical allows Poisson's ratios in isotropic materials in the range -1 to $1 / 2$. Common materials exhibit a Poisson's ratio from $1 / 4$ to nearly $1 / 2$. We have prepared materials with a Poisson's ratio as small as -0.8. Deformation mechanisms in these materials include relative rotation of micro-elements, and non-affine micro-deformation. The relation between properties and structure is exploited to prepare viscoelastic composites with high stiffness combined with high damping. Generalized continuum theories exist with more freedom than classical theory. For example, in Cosserat elasticity there are characteristic lengths as additional engineering elastic constants. Recent experimental work discloses a variety of cellular and fibrous materials to exhibit such freedom, and the characteristic lengths have been measured. In hierarchical solids structural elements themselves have structure. Several examples of natural structural hierarchy are considered, with consequences related to optimality of material properties.
\end{abstract}

\title{
1 Introduction
}

For many purposes it is expedient to make use of continuum representations of materials, all of which actually have structure. The various length scales in the structure have various consequences as shown in Fig. 1.1. In composites of simple structure it is possible to predict or to bound the behavior of the composite from the behavior of the constituents [see, e.g. 1.1, 1.2]. There is a great variety of possible material micro-structure. We consider the question of the extent to which this structure has consequences in the behavior of a material viewed as a continuum. One theme to be explored is that of extremal properties. In section 2, negative Poisson's ratios are considered; in section 3, viscoelastic composites with high stiffness and damping; and in section 5, the optimization of compressive strength in low-density cellular solids. The structural concept underlying negative Poisson's ratio is that of non-affine deformation, for example the unfolding of cells in a cellular solid. Non-affine deformation on the composite scale, combined with atomic scale damping mechanisms also may be used to achieve composites with high damping and high stiffness. The structural concept underlying cellular solids with optimal compressive strength is that of structural hierarchy. There are further aspects of material behavior in addition to optimization of particular characteristics. Given certain types of micro-structure, qualitatively new phenomena may occur in the mechanics of materials. Several of these have been anticipated in the development of generalized continuum representations such as Cosserat elasticity, which has characteristic length scales. In a Cosserat solid the points in the continuum have the freedom to rotate as well as to translate. Cosserat elasticity is discussed in section 4, from an experimental perspective. A variety of experimental results are given. One consequence of Cosserat elasticity is an enhancement of the toughness of a material, even if it has no ductile constituents. Theoretical results of Cosserat elastic constants derived from structural considerations are reviewed. Fibrous, cellular and laminated materials are predicted to exhibit observable characteristic lengths, but particulate materials are predicted to exhibit characteristic lengths of zero, corresponding to classical elasticity. 


\section{Control of Poisson's ratio}

Poisson's ratio of a solid is defined as the lateral contraction strain divided by the longitudinal extension strain in a simple tension experiment. Poisson's ratio, usually denoted by $v$, is positive in almost all known materials. For isotropic materials (those in which the properties are independent of direction), it may be shown via energy arguments in the theory of elasticity [2.1] that $-1 \leq v \leq 1 / 2$.

The relationship between shear modulus $\mathrm{G}$, bulk modulus $\mathrm{K}$, and Poisson's ratio $\mathrm{v}$ for isotropic materials is

$\mathrm{G}=\frac{3 \mathrm{~K}(1-2 v)}{2(1+v)}$.

Many common materials have a Poisson's ratio close to 1/3. Rubbery materials have values approaching 1/2; they readily undergo shear deformations but resist volumetric (bulk) deformation, so $\mathrm{G}<<\mathrm{K}$ above. Some textbooks can still be found which categorically state that Poisson's ratios less than zero are unknown; nevertheless there are in fact a number of examples of negative Poisson's ratio solids. Such solids become fatter in cross section when stretched. A solid with $v \approx$ -1 would be the opposite of rubber: difficult to shear but easy to deform volumetrically: $\mathrm{G}>>\mathrm{K}$. Foams and honeycombs have two length scales, the size of the cell and the size of the rib. Those two scales confer the freedom to control material properties via cell shape. The continuum aspects corresponding to the structures considered in this section are examined in section 4.

Two dimensional honeycombs with inverted cells (Fig. 2.1b; compare with conventional honeycomb with convex hexagonal cells in Fig. 2.1a) were reported with negative Poisson's ratios in the honeycomb plane [2.2-2.5], and these honeycombs have been recently analyzed [2.6,2.7]. Honeycombs are sufficiently simple that the Poisson effect can be easily visualized; moreover, demonstration models can be easily made from paper. Macroscopic structures in two or three dimensions consisting of rods, springs, and sliders, were devised to give a negative Poisson's ratio [2.5]; these structures have an inverted characteristic similar to the honeycombs. Structures of the above type require some form of individual assembly.

Foam materials (three-dimensional cellular solids) with a negative Poisson's ratio as small as -0.7 were developed [2.8] in which an inverted or re-entrant cell structure was achieved by isotropic permanent volumetric compression of a conventional foam, resulting in microbuckling of the cell ribs. A schematic diagram of a typical tetrakaidecahedral cell of a normal foam is shown in Fig. 2.1c, and a re-entrant cell in Fig. 2.1d. Polymer foams which exhibit a softening point [2.8,2.9], ductile metallic foams [2.8,2.9], and thermosetting polymer foams [2.9] can be prepared with a negative Poisson's ratio. The method for open cell polymer foams is to compress a foam block triaxially in a mold, heat above the softening point, and cool. The method for ductile metal foam is to apply small increments of plastic deformation in each of three orthogonal directions. The method for thermosetting foam is to apply triaxial compression at an appropriate time during the foaming process. Representative polymer foam structure is shown for normal foam in Fig. 2.2a and in reentrant foam in Fig. 2.2b. Poisson's ratio depends on strain [2.10,2.11]; the range for negative Poisson's ratio is larger in the polymer than in the metal foams as a result of the plastic hinge formation which occurs in the ribs of the metal foams. In these structures and materials, the negative Poisson's ratio arises from the unfolding of the re-entrant cells, and isotropy can be achieved along with the negative Poisson's ratio. They are true materials in that no individual assembly is required.

Negative Poisson's ratios can occur in certain directions in several anisotropic materials. For example, certain anisotropic microcellular foams exhibit an unintentional negative Poisson's ratio in some directions $[2.12,2.13]$. These are anisotropic materials, therefore the bounds on Poisson's ratio are wider $(-\infty<v<\infty)$ than in the isotropic case; indeed Poisson's ratios smaller than -1 have been reported. The causal mechanism appears to be associated with tilting of particulates linked by microfilaments. Negative Poisson's ratios have been theoretically analyzed in anisotropic fibrous composites. It is theoretically possible in some laminates to achieve a negative Poisson's ratio as small as -0.21 in the direction perpendicular to the layers by control of the stacking sequence [2.14]. Negative Poisson's ratio in other laminates can be achieved in some directions in the plane 
of the laminate, again by control of the stacking sequence [2.15,2.16]. In these composites there is a high degree of anisotropy and the negative Poisson's ratio only occurs in some directions; in some cases only over a narrow range of orientation angle between the applied load and the fibers. Other anisotropic systems which may exhibit negative Poisson's ratio are some single crystals such as iron pyrites [2.17], though random aggregates of crystals almost always have a positive Poisson's ratio [2.18]

Laminate structures have been presented which give rise to intentional negative Poisson's ratios combined with mechanical isotropy in two dimensions (Fig. 2.3) or in three dimensions [2.19]. These laminates have structure on several levels of scale; they are hierarchical and have many length scales (see Fig. 1.1 and section 5). One can achieve, by appropriate choice of constituent properties, Poisson's ratios approaching the lower limit of -1 .

Negative Poisson's ratios are counterintuitive. It is of interest to consider causes. Causes must arise in the microstructure, since the continuum theories of elasticity do not specify particular values of Poisson's ratio. A solid containing atoms interacting by central forces, in which the atoms move in an affine manner (locally equivalent to a strain combined with a rotation) under deformation, exhibits a Poisson's ratio of $1 / 4$ [2.20,2.21]. This result is originally due to S. D. Poisson. Most ordinary materials have Poisson's ratio typically close to $1 / 3$, so at least one of these assumptions must not apply to them. In negative Poisson's ratio foams as in conventional ones, bending of cell ribs occurs. Such bending is insufficient as a causal mechanism for the negative Poisson's ratio [2.22] contrary to an earlier suggestion [2.23]. Negative Poisson's ratios cannot be achieved via the bending or twisting rigidity of the cell ribs alone [2.24]. Non-affine (locally inhomogeneous) deformation by itself can give rise to the negative Poisson's ratio effects [2.24]. Other possible causes are pre-strain in the lattice structure [2.24] or a chiral structure [2.24,2.25]; see discussion in $\$ 4$. Several illustrative models involving truss elements with sliders, and hypothetical granular structures, have been presented [2.26, 2.27].

In the re-entrant foams and honeycombs, inhomogeneous, non-affine deformation is manifested as an unfolding of the cells as the entire structure is extended. Such deformation has been experimentally observed in foams [2.28]. The structure of some of the crystalline materials, notably $\alpha$-cristobalite and arsenic, suggests that non-affine deformation occurs on the atomic scale as well.

The composites of Milton [2.19] offer the intriguing possibility of stiff materials with a negative Poisson's ratio. The stiffness of one the two constituents must be at least 25 times greater than that of the other constituent to obtain a Poisson's ratio less than zero. A Poisson's ratio approaching -1 requires constituents which differ even more in stiffnesses: one phase becomes very soft, tending to 'empty space' in its properties. Another intriguing possibility is that of creating stiffer negative Poisson's ratio materials by design on the molecular scale [2.29]. Further review of the area of negative Poisson's ratio materials is provided in [2.30].

As for possible applications of negative Poisson's ratio foams, they may find use in absorbing sound and vibration by virtue of resonance of the ribs [2.31], unusual fasteners [2.32], toughened foams, abrasion-resistant sponge, and damage-resistant core for sandwich panels. 


\section{$3 \quad$ Viscoelastic composites}

In viscoelastic materials the material properties depend on time or frequency, and mechanical energy is dissipated in deformation. The constitutive equation for linear viscoelasticity is the Boltzmann superposition integral. Here $t$ is time and $\tau$ is a time variable of integration.

$\sigma(\mathrm{t})=\int_{0}^{\mathrm{t}} \mathrm{E}(\mathrm{t}-\tau) \frac{\mathrm{d} \varepsilon}{\mathrm{d} \tau} \mathrm{d} \tau$

In the frequency domain, the modulus may be regarded as a complex number dependent on frequency: $\mathrm{E}^{*}=\mathrm{E}^{\prime}+\mathrm{iE}$ ". The ratio of the imaginary part $\mathrm{E}^{\prime \prime}$ to the real part $\mathrm{E}^{\prime}$ is of interest physically and is referred to as the loss tangent.

$\tan \delta=\mathrm{E}^{\prime \prime} / \mathrm{E}^{\prime}$

Viscoelastic materials are of use in the damping of vibration and in the absorption of sound waves. It is desirable in some applications to use materials which are stiff enough to carry out a structural role, and which also exhibit significant mechanical damping. Most stiff materials, however, are low in damping and most high-damping materials are compliant. Properties of some representative materials are shown in Fig. 3.1.

Non-affine deformation, in which the strain field is highly inhomogeneous on the scale of micro-structural elements was considered above in the context of materials with a negative Poisson's ratio. Non affine deformation in composite materials permits one to simultaneously achieve high stiffness and high loss [3.1]. Such deformation occurs in negative Poisson's ratio materials and also in Reuss-type laminates. Reuss laminates exhibit minimal stiffness for a given volume fraction of the stiff phase, however a stiff Reuss composite can be made if the volume fraction of the compliant phase is made very small.

We use stiffness-loss maps to examine the consequences of changes in constituent properties with the aim of developing approaches for producing composite materials with high stiffness and high loss. The maps are constructed from the equations for the stiffness of an elastic composite, with the aid of the dynamic correspondence principle of linear viscoelasticity [3.2,3.3]. Each elastic modulus is replaced with a complex dynamic modulus in the analysis. In particular, for elastic phases, the Voigt (or uniform strain) relation is

$\mathrm{E}_{\mathrm{c}}=\mathrm{E}_{1} \mathrm{~V}_{1}+\mathrm{E}_{2} \mathrm{~V}_{2}$,

in which $\mathrm{E}_{\mathrm{c}}, \mathrm{E}_{1}$ and $\mathrm{E}_{2}$ refer to the Young's modulus of the composite, phase 1 and phase 2 , and $\mathrm{V}_{1}$ and $V_{2}$ refer to the volume fraction of phase 1 and phase 2 with $V_{1}+V_{2}=1$. We apply the correspondence principle to convert the elastic relations to a viscoelastic relation (for loading which is sinusoidal in time) by replacing the Young's moduli $\mathrm{E}$ by the complex modulus $\mathrm{E}^{*}(\mathrm{i} \omega)$ or $\mathrm{E}^{*}=$ $E^{\prime}+i E^{\prime \prime}$, in which $\omega$ is the angular frequency of the harmonic loading. This procedure gives

$\mathrm{E}_{\mathrm{c}}^{*}=\mathrm{E}_{1}^{*} \mathrm{~V}_{1}+\mathrm{E}_{2}{ }^{*} \mathrm{~V}_{2}$,

with the loss tangent $\tan \delta=E^{\prime \prime} / E^{\prime}$. Similar analysis may be conducted for the Reuss structure and for other composite structures [3.4].

The curves for the Voigt and Reuss composites enclose a region in the map. Although the Voigt and Reuss relations bound the elastic stiffness for given volume fraction, they do not necessarily bound the viscoelastic behavior in a stiffness loss map. Bounds for the complex bulk modulus have been presented [3.5]. We remark that the Voigt and Reuss curves appear almost identical to the Hashin-Shtrickman stiffness bounds on a stiffness-loss map [3.1] even though they can be very different when plotted as functions of volume fraction; moreover they do not differ much from the true bounds. Fig. 3.2 shows the effect of increasing the stiffness of the stiff phase, from $200 \mathrm{GPa}$, corresponding to steel, to $400 \mathrm{GPa}$, corresponding to tungsten. For both, $\tan \delta$ is assumed to be 0.001 , a value representative of results reported in the literature. The more compliant phase is assumed to have $\mathrm{E}=31 \mathrm{GPa}$, and $\tan \delta=0.12$. Observe that use of a stiffer stiff phase facilitates achievement of a composite which is both stiff and lossy.

Experimental studies, using an instrument [3.6] capable of measurements over ten decades $\left(10^{-6} \mathrm{~Hz}\right.$ to $\left.10^{4} \mathrm{~Hz}\right)$, on a laminate of tungsten and indium-tin alloy disclose a stiffness $\mathrm{E}=161$ 
GPa with $\tan \delta=0.096$ achieved at $0.1 \mathrm{~Hz}$, and $\mathrm{E}=129 \mathrm{GPa}$ with $\tan \delta=0.2$ at $0.01 \mathrm{~Hz}$ [3.4]. This behavior is well above the diagonal line in Fig. 3.1 and represents a very unusual combination of stiffness and damping.

The potential benefit of such materials is a reduction in damage to vehicles due to vibration and metal fatigue, a reduction in operator fatigue, reduction in noise due to machinery, reduction in disturbances to mobile electronic equipment, and improved performance due to reductions in weight.

\section{Cosserat elasticity Constitutive equations}

The classical theory of elasticity is presently used in engineering analyses of deformable objects at small strain. Other continuum theories are available, all mathematically consistent, for linear isotropic materials. Some have more freedom, and some have less freedom than classical elasticity. Therefore a discrimination among them is to be made by experiment.

The early uniconstant elasticity theory of Navier is based upon the assumption that forces act along the lines joining pairs of atoms and are proportional to changes in distance between them [4.1]. The constitutive equation is as follows.

$$
\sigma_{\mathrm{kl}}=\mathrm{G} \varepsilon_{\mathrm{rr}} \delta_{\mathrm{kl}}+2 \mathrm{G} \varepsilon_{\mathrm{kl}}
$$

$\sigma$ is stress, $\varepsilon$ is strain, and $G$ is an elastic constant, the shear modulus. This uniconstant theory was accepted by Navier, Cauchy, Poisson, and Lamé during the early days of the theory of elasticity. The theory contains less freedom than the classical theory of elasticity now in common use. There is no length scale in uniconstant elasticity.

Classical isotropic elasticity [4.2,4.3], has the following constitutive equation, in which there are the two independent elastic constants $\lambda$ and $G$, the Lamé constants.

$$
\sigma_{\mathrm{kl}}=\lambda \varepsilon_{\mathrm{rr}} \delta_{\mathrm{kl}}+2 \mathrm{G} \varepsilon_{\mathrm{kl}}
$$

The Poisson's ratio $v=\lambda / 2(\lambda+G)$ is restricted by energy considerations to have values in the range from -1 to $1 / 2$. There is no length scale in classical elasticity.

The Cosserat theory of elasticity [4.4] incorporates a local rotation of points as well as the translation assumed in classical elasticity; and a couple stress (a torque per unit area) as well as the force stress (force per unit area). The force stress is referred to simply as 'stress' in classical elasticity in which there is no other kind of stress. The idea of a couple stress can be traced to Voigt [4.5] during the early development of the theory of elasticity. More recently, theories incorporating couple stresses were developed using the full capabilities of modern continuum mechanics [4.64.10]. Eringen [4.9] incorporated micro-inertia and renamed Cosserat elasticity micropolar elasticity. In the isotropic Cosserat solid there are six elastic constants, in contrast to the classical elastic solid in which there are two, and the uniconstant material in which there is one. The constitutive equations for a linear isotropic Cosserat elastic solid are, in the symbols of Eringen [4.9]:

$$
\begin{aligned}
& \sigma_{\mathrm{kl}}=\lambda \varepsilon_{\mathrm{rr}} \delta_{\mathrm{kl}}+(2 \mu+\kappa) \varepsilon_{\mathrm{kl}}+\kappa \mathrm{e}_{\mathrm{klm}}\left(\mathrm{r}_{\mathrm{m}}-\phi_{\mathrm{m}}\right) \\
& \mathrm{m}_{\mathrm{kl}}=\alpha \phi_{\mathrm{r}, \mathrm{r}} \delta_{\mathrm{kl}}+\beta \phi_{\mathrm{k}, 1}+\gamma \phi_{1, \mathrm{k}}
\end{aligned}
$$

The usual summation convention for repeated indices is used throughout, as is the comma convention representing differentiation with respect to the coordinates. $\sigma_{\mathrm{kl}}$ is the force stress, which is a symmetric tensor in equations 1 and 2 but it is asymmetric in Eq. $4.3 . \mathrm{m}_{\mathrm{kl}}$ is the couple stress, $\varepsilon_{\mathrm{kl}}=\left(\mathrm{u}_{\mathrm{k}, \mathrm{l}}+\mathrm{u}_{1, \mathrm{k}}\right) / 2$ is the small strain, $\mathrm{u}_{\mathrm{k}}$ is the displacement, and $\mathrm{e}_{\mathrm{klm}}$ is the permutation symbol. The microrotation $\phi_{\mathrm{k}}$ in Cosserat elasticity is kinematically distinct from the macrorotation $\mathrm{r}_{\mathrm{k}}=$ $\left(\mathrm{e}_{\mathrm{klm}} \mathrm{u}_{\mathrm{m}, 1}\right) / 2$ obtained from the displacement gradient. Components of stress and couple stress on a differential element of a Cosserat solid, and the corresponding increments of force and moment on the structural elements of a real material are shown in Fig. 4.1.

In three dimensions, the isotropic Cosserat elastic solid is described by six elastic constants $\lambda, \mu, \alpha, \beta, \gamma$, and $\kappa$. Symbols used by various authors were compared by Cowin [4.11]. The 
following technical constants derived from the tensorial constants are more beneficial in terms of physical insight. These are [4.9,4.12]:

Young's modulus $E=(2 \mu+\kappa)(3 \lambda+2 \mu+\kappa) /(2 \lambda+2 \mu+\kappa)$,

shear modulus $\mathrm{G}=(2 \mu+\kappa) / 2$,

Poisson's ratio $v=\lambda /(2 \lambda+2 \mu+\kappa)$,

characteristic length for torsion $\mathrm{I}_{\mathrm{t}}=[(\beta+\gamma) /(2 \mu+\kappa)]^{1 / 2}$,

characteristic length for bending $I_{b}=[\gamma / 2(2 \mu+\kappa)]^{1 / 2}$,

coupling number $N=[\kappa / 2(\mu+\kappa)]^{1 / 2}$, and

polar ratio $\Psi=(\beta+\gamma) /(\alpha+\beta+\gamma)$.

The Cosserat characteristic lengths are of particular interest. They are in contrast to classical elasticity which has no length scale. When the Cosserat elastic constants $\alpha, \beta, \gamma, \kappa$ vanish, the solid becomes classically elastic. The case $\mathrm{N}=1$ (its upper bound) is known as 'couple stress theory' [4.8. 4.13]. $\mathrm{N}=1$ corresponds to $\kappa \rightarrow \infty$, a situation which is permitted by energetic considerations, as is incompressibility in classical elasticity. The case $\kappa=0$ corresponds to a decoupling of the rotational and translational degrees of freedom. Although some theoreticalwriters choose to solve problems for this case since the analysis is simpler, the limit $\kappa \rightarrow 0$ presents physical difficulties [4.14].

\section{Predicted behavior}

Materials obeying different continuum theories can be distinguished since predicted behavior is different. Some of these differences in behavior can be used in the design of experiments to explore the generalized continuum aspects of materials. The discussion below is for the most part restricted to isotropic materials since their behavior is simpler.

The uniconstant theory predicts a Poisson's ratio of $>$ for all isotropic materials. Since most common isotropic materials exhibit a Poisson's ratio close to <, the uniconstant theory was rejected based on experimental measurements of Poisson's ratio. The issue was not decided until well after the introduction of the theories since decisive experiments were difficult to perform in the late 1800 's.

Classical elasticity is, according to its name, the currently accepted theory of elasticity. The following behavior is predicted.

(i) The rigidity of circular cylindrical bars of diameter $\mathrm{d}$ in tension is proportional to $\mathrm{d}^{2}$; in bending and torsion, the rigidity is proportional to $\mathrm{d}^{4}$.

(ii) The wave speed of plane shear waves and dilatational waves in an unbounded medium is independent of frequency.

(iii) There is no length scale in classical elasticity, hence stress concentration factors for holes or inclusions in an infinite domain under a uniform stress field depend only on the shape of the inhomogeneity, not on its size.

(iv) Poisson's ratio $v$ for isotropic materials can have values in the range $-1 \leq v \leq \mathfrak{\alpha}$.

Cosserat or micropolar elasticity has the following consequences in isotropic materials.

(i) A size-effect is predicted in the torsion of circular cylinders of Cosserat elastic materials. Slender cylinders appear more stiff then expected classically [4.12]; Fig. 4.2. A similar size effect is also predicted in the bending of plates [4.12] and of beams [4.15]; Fig. 4.3. No size effect is predicted in tension.

(ii) The stress concentration factor for a circular hole, is smaller than the classical value, and small holes exhibit less stress concentration than larger ones [4.16].

(iii) The wave speed of plane shear waves and dilatational waves in an unbounded Cosserat elastic medium is independent of frequency as in the classical case. The speed of shear waves depends on frequency in a Cosserat solid [4.9]. A new kind of wave associated with the microrotation is predicted to occur in Cosserat solids. 
(iv) The range in Poisson's ratio is from -1 to +0.5 , the same as in the classical case [4.12].

Chiral Cosserat solids

New phenomena are predicted in Cosserat solids which lack a center of symmetry. These solids are called noncentrosymmetric or chiral materials. A rod under tensile load deforms in torsion [4.17]. Wave speed for transverse circularly polarized waves depends on the sense of polarization. This leads to rotation of the principal plane of elliptically polarized transverse waves [4.18]. Crystalline materials such as sugar are chiral on an atomic scale, and composites withhelical inclusions or spiraling fibers are chiral on the microstructural level.

Chirality has no effect on the classical elastic properties of a material. To demonstrate this, consider the tensorial Hooke's law for classical elasticity:

$\sigma_{\mathrm{ij}}=\mathrm{C}_{\mathrm{ijkl}} \varepsilon_{\mathrm{kl}}$,

in which $\sigma_{\mathrm{ij}}$ is stress, $\varepsilon_{\mathrm{kl}}$ is strain and $\mathrm{C}$ is the elastic modulus tensor.

The transformation law for the modulus tensor under coordinate changes is

$\mathrm{C}_{\mathrm{ijkl}}{ }^{\prime}=\mathrm{a}_{\mathrm{im}} \mathrm{a}_{\mathrm{jn}} \mathrm{a}_{\mathrm{ko}} \mathrm{a}_{\mathrm{In}} \mathrm{C}_{\text {mnop }}$.

For an inversion, the transformation matrix is just the negative of a Kronecker delta

$\mathrm{a}_{\mathrm{im}}=-\delta_{\mathrm{im}}$.

So the classical elastic modulus tensor is unchanged by chirality:

$\mathrm{C}_{\mathrm{ijkl}}{ }^{\prime}=(-1)^{4} \mathrm{C}_{\mathrm{ijkl}}=\mathrm{C}_{\mathrm{ijkl}}$.

Since the constitutive equation for Cosserat elasticity (allowing anisotropy) has higher order tensors, any lack of invariance to inversion will have an effect. Therefore the constitutive equation for a Cosserat solid which is directionally isotropic but chiral, will contain extra terms [4.17]; there are 9 elastic constants for such a chiral solid in contrast to the 6 which describe the fully isotropic Cosserat solid.

\section{Experiment designs}

Uniconstant and classical solids may be distinguished by measurements of Poisson's ratio. Such experiments done in the last century led to the adoption of what is now called classical elasticity.

Cosserat solids may be characterized via size effects in rigidity. Exact analytical solutions for size effects form the basis of a variety of experiments for the characterization of Cosserat solids. For example, Gauthier and Jahsman [12] give $\Omega$, the ratio of rigidity to its classical value, for cylindrical bending of a plate,

$\Omega=1+24 \frac{\mathrm{I}^{2}(1-v)}{\mathrm{h}^{2}}$

with $\mathrm{h}$ as the plate thickness. The length parameter of Gauthier and Jahsman was converted into the characteristic length for bending defined above. In plate bending, the anticlastic curvature due to the Poisson effect is constrained, in contrast to beam bending.

For torsion of a circular cylinder [12], the ratio $\Omega$ of rigidity to its classical value is

$\left.\Omega=1+6(I \mathrm{t} / \mathrm{r})^{2}[1-4 \Psi \mathrm{X} / 3) /(1-\Psi \mathrm{X})\right]$,

with $\mathrm{r}$ as the rod radius, $\Psi=(\beta+\gamma) /(\alpha+\beta+\gamma)$ and $X=\mathrm{I}_{1}(\mathrm{pr}) / \mathrm{pr} \mathrm{I}_{0}(\mathrm{pr})$, and $\mathrm{p}^{2}=2 \kappa /(\alpha+\beta+\gamma)$. $\mathrm{I}_{1}$ and $\mathrm{I}_{0}$ are the modified Bessel functions of the first kind. A special case of interest, referred to as 'couple stress elasticity', is for $\mathrm{N}=1(\kappa \rightarrow \infty)$ in which the [] bracket in equation 4.10 becomes unity. If the rod diameter is large, the corresponding rigidity ratio is $\Omega \approx 1+6(\mathrm{l} t / \mathrm{r})^{2}$.

For bending [4.15] of a circular section rod of radius $r$, the rigidity ratio $\Omega$ is

$\Omega=1+8\left(\mathrm{I}_{\mathrm{b}} / \mathrm{r}\right)^{2}\left(1-(\beta / \gamma)^{2}\right)+\left[\left(8 \mathrm{~N}^{2}(\beta / \gamma+\mathrm{v})^{2} /\left(\zeta(\delta \mathrm{a})+\mathrm{N}^{2}(1-\mathrm{v})\right)(1+\mathrm{v})\right]\right.$

with $\zeta(\delta \mathrm{r})=(\delta \mathrm{r})^{2}\left[\left(\delta \mathrm{r}_{0}(\delta \mathrm{r})-\mathrm{I}_{1}(\delta \mathrm{r})\right) /\left(\delta \mathrm{r} \mathrm{I}_{0}(\delta \mathrm{r})-2 \mathrm{I}_{1}(\delta \mathrm{r})\right)\right]$, and $\delta=\mathrm{N} / \mathrm{I}_{\mathrm{b}}$. 
Data may be plotted as $\Omega$ vs radius; however one may also plot rigidity divided by the square of the diameter vs. the square of the diameter for torsion (Fig. 4.2) and bending (Fig. 4.3). They are not stress strain curves. The utility of such plots is that the characteristic lengths can be extracted from the intercept of the extrapolated straight portion of the curve upon the ordinate.

In classical elasticity, the torsion size effect plot is a straight line through the origin with slope proportional to the shear modulus G; similarly the slope of the size effect plot for bending gives Young's modulus E. Cosserat elastic constants for a material may be extracted via comparison of the experimental curve with the theoretical one. The characteristic lengths are obtained by intercepts as indicated in the figures. Either graphical or numerical methods may be used. The shape of the torsion plot is then used to extract the coupling number $\mathrm{N}$. A large value of $\mathrm{N}$ (the upper bound is 1) leads to a large apparent stiffening for slender specimens. The shape of the torsion plot in the vicinity of the origin is used to determine $\Psi$; this is difficult since it requires very thin specimens. We have used a numerical algorithm to minimize the mean-square deviation between the experimental data and the theoretical graphs, to extract the elastic constants.

\section{Experimental results}

Experimental results have been reported for a variety of materials. For example, metals such as steel and aluminum behave entirely classically [4.19], with I $=0$, as might be expected from the long history of successful use of classical elasticity. Gauthier and Jahsman [4.12] fabricated a particulate composite in the hope that it would manifest size effects consistent with Cosserat elasticity, however no such effects were found.

Human compact bone was found to be Cosserat elastic [4.20-4.22] with characteristic lengths on the order of the size of the osteons, which are fiber-like structures, about $0.2 \mathrm{~mm}$ in diameter, within bone. An amorphous polymer, PMMA, studied as a control, proved to behave classically. Ascenzi et. al. [4.23] recently reported high shear moduli in tests upon wet single human femoral osteons of various fiber architectures. These were interpreted in terms of a Cosserat view [4.24]. As shown in Fig. 4.4, the apparent shear modulus of bone specimens of different diameter, in torsion, depends upon the diameter. Large size effects are observed. Although some writers consider effects associated with generalized continuum mechanics to be "higher order terms" which are supposed to be invariably small, these experiments show that for certain materials, large effects may be observed; in bone, a factor greater than six in effective stiffness.

Cosserat elastic effects were also observed experimentally in a variety of foam materials [4.25,4.26]. The Cosserat curves in Fig. 4.2 and 4.3 are representative of the sort of data obtained. Inferred characteristic lengths were on the order of the size of the cells in the foams for most foams studied. In the case of an isotropic foam [4.26] it was possible to conduct cross-checks similar to the relation among Young's and shear moduli and Poisson's ratio in classical elasticity. The crosschecks, which depend on the fact that some Cosserat elastic constants influence the shape of both the bending and torsion size effect plots, were satisfied. Syntactic foam [4.26], which consists of stiff hollow glass microspheres in an epoxy matrix, proved to behave nearly classically, in contrast to other foams. The microspheres behave as stiff particles. Classical behavior of particulate materials is consistent with generalized composite theory as described below.

One may experimentally explore materials as generalized continua via study of the distribution of strain in deformed objects, in contrast to the above study of size effects in which the rigidity, a global quantity, is measured. The distribution of strain in a bar of rectangular cross section under torsion was analyzed [4.27] and the analysis applied to deformation of square bars of bone [4.28] which was earlier characterized by size effect methods [4.20,4.21]. The surface strain does not vanish at the corner of the cross section, in contrast to the case of classical elasticity. The presence or absence of corner strain may be illustrated in holographic interferometry images [4.29] or in macroscopic observation of a notch in the corner of a flexible bar of foam [4.30]. Moreover the strain field around a stress concentrator such as a circular hole is predicted to differ depending on whether the solid is classical [4.2,4.3] or Cosserat [4.9, 4.13,4.16].

\section{Predictive power}


The experimental evaluation of materials as Cosserat solids is useful if it permits one to predict stresses and strains under conditions which differ from those in the experiments used to find the elastic constants.

Predictive power of Cosserat elasticity has been studied experimentally. For example, sizeeffect studies of a dense polyurethane foam yielded the six Cosserat elastic constants [4.25]. Holographic study of a square section bar of the same foam under torsion disclosed deformation at the corner [4.29] which was predicted by Cosserat elasticity. This is in contrast to a similar bar of amorphous polymer (PMMA) which is classical as demonstrated via size effects, and which exhibited no deformation at the corner, in agreement with predicted behavior.

In human compact bone, Cosserat elastic constants (based on isotropic theory) [4.21] were used to predict the strain distribution around a hole in a strip under tension. Reasonable agreement with experiment was found [4.31] even though bone is anisotropic. Cosserat elastic constants, derived from size effect studies, were used to predict strain distributions in a square bar under torsion. Nonclassical strain distributions were in fact observed [4.28] and agreed well with the distributions predicted by Cosserat elasticity.

\section{Cosserat composite theory}

Several authors have developed composite theoretical approaches to the determination of Cosserat elastic constants from consideration of the microstructure and from the constituent elastic properties [4.32-4.35]. Fibrous and laminated structures give rise to Cosserat elastic behavior while particulate morphologies give zero Cosserat characteristic length. These theoretical results are in harmony with the above experiments: bone is fibrous but a model particulate material [4.12] disclosed no Cosserat effects.

We remark that the recent use of homogenization procedures in the theoretical study of composite mechanics gives the classical elastic properties, with no unexpected freedom. Since the extra freedom is not injected into the theory in the initial stages, it will not appear in the results.

\section{Significance}

The significance of Cosserat elasticity is that it predicts strain distributions and resultants which, for a certain class of materials, is in better accord with reality as revealed by experiment, than classical elasticity. One practically important aspect of the altered distributions is that strain tends to "spill over" from regions which classically would have high strain to those which classically would have low strain. The result is a toughening effect. It is interesting to observe that although classical elasticity has no length scale, the units for toughness, $\mathrm{MPa} \sqrt{\mathrm{m}}$, incorporate a length dimension. In many materials, the connection between the length dimension in toughness with structural length scales is rather indirect. However, in foams, formulae for the toughness depend on the size of the foam cells in an explicit way [2.7]. In the structural view, the cause of both Cosserat elasticity and toughness may be sought in the largest length scale in the material, as depicted in Fig. 1.1.

\section{$5 \quad$ Structural hierarchy}

Structural hierarchy refers to a solid or structure which contains structural elements which themselves have structure (see, e.g., [5.1]). The hierarchical order of a structure or a material may be defined as the number $n$ of levels of scale with recognized structure. $n=0$ corresponds to a material viewed as a continuum for the purpose of analysis of physical properties; $n=1$ (first order) could represent a latticework of continuous ribs or the atomic lattice of a crystal. Macroscopichierarchical frameworks can be traced back at least to Eiffel's design for his tower [5.2] and of Eiffel's bridges such as the Garabit Viaduct. The role of multiple length scales in relation to the other structural issued examined here, is illustrated in Fig. 1.1.

Hierarchical honeycomb and foam materials exhibit substantial improvements in compressive strength in comparison with conventional materials of hierarchical order 1 . The demonstration of the strength enhancement may be done [5.1] with the aid of the governing equations for the conventional cellular solids [5.3]. As shown in Fig. 5.1, strength of hierarchical foam and honeycomb can be greatly enhanced by the use of structural hierarchy. Some preliminary confirming experimental results [5.1] demonstrated strength improvement in a second order 
honeycomb. The physical mechanism for the improved strength is the suppression of buckling in the hierarchical structure.

Human compact bone is a natural composite which exhibits a rich hierarchical structure. The largest structure is that of the whole bone. On the microstructural level are the osteons, which are large ( $0.2 \mathrm{~mm}$ dia.) hollow fibers composed of concentric lamellae and of pores. The lamellae are built of fibers, and the fibers contain fibrils. At the ultrastructural level (nanoscale) the fibers are a composite of the mineral hydroxyapatite and the protein collagen. There is a relation between bone structure and various physical properties. Stiffness [5.4] of bone arises from the composite structure of mineral microcrystals and protein (principally collagen) fibers. The cement lines as weak interfaces between osteons (large fibers) impart a degree of toughness [5.5] to bone as well as viscoelasticity [5.6]. As for pores, the lacunae are ellipsoidal pores which provide space for the osteocytes, the living cells of bone. The bone cells at this level of scale permit bone tissue to remodel its structure in response to prevailing stresses [5.7]. Haversian canals are cylindrical pores which contain blood vessels which nourish the tissue. Canaliculi are very fine channels radiating from the lacunae. Mechanical stress in bone due to physical activity is considered to be important in pumping nutrients through these channels [5.8]. The pore structure of bone is essential in maintaining its viability and consequently its ability to adapt to mechanical stress. A two level hierarchical analytical model [5.9] has been used to predict anisotropic elasticity of bone; it successfully modeled how bone stiffness depends on the orientation of applied stress with respect to the osteon axis. Other materials of biological origin, such as wood and tendon, also have a rich hierarchical structure.

\section{Discussion and conclusions}

The role of the structural and continuum views in the mechanical freedom in solids has been explored. It has been expedient in that vein to consider the various structural length scales in the material. It was known for a long time, based on continuum concepts, that negative Poisson's ratios were energetically permissible. Early structural studies of the question of elastic constants did not incorporate enough freedom to anticipate such a possibility. The first developments of negative Poisson's ratio materials have come from the experimental side, and have been linked to the structural concept of non-affine deformation, which has also been used in developing high performance viscoelastic materials.

Degrees of freedom in the mechanical behavior of materials are not always anticipated in optimization schemes. For example, one may derive the continuum properties of a composite via a homogenization procedure, and obtain a structure which gives the maximal stiffness by an optimization procedure. Such a procedure will not reveal freedom such as that represented by Cosserat elasticity unless that freedom is incorporated in the analysis.

As for Cosserat elasticity, the possibility of unusual phenomena was anticipated from the continuum side, but only recently have these phenomena been explored experimentally. Cosserat effects have been demonstrated in a variety of materials, and the Cosserat theory found to have the ability to make predictions of nonclassical effects in geometries different from those used to determine the Cosserat elastic constants. Further detail as well as methods for comparing different generalized continuum theories have been presented in a prior review [4.32]. Future development may include designing material microstructure, inspired by Cosserat concepts, to achieve high toughness.

As for structural hierarchy, such structure is a ubiquitous feature of biological materials. Concepts of structural hierarchy have been used to optimize known material properties such as compressive strength or Poisson's ratio. Such optimization is to be distinguished from the creation of new and unexpected phenomena. We anticipate the possibility of such developments in the future.

\section{References}

1.1. Hashin Z., and Shtrickman, S., "A variational approach to the theory of the elastic behavior of multiphase materials", J. Mech. Phys. Solids, 11, 127-140, 1963. 
1.2. Hashin, Z., "Analysis of composite materials- a survey", J. Applied Mechanics, 50, 481-505, 1983.

2.1 Fung, Y. C., Foundation of Solid Mechanics, Prentice-Hall, Englewood, NJ, p.353, 1968.

2.2 Schajer, G. S. and Robertson, C. I., "Mechanical behaviour of cellular structures", project report, Pembroke college, Cambridge University, 1974.

2.3 Gibson, L. J., Ashby, M. F., Schajer, G. S. and Robertson, C. I., "The mechanics of two dimensional cellular solids", Proc. Royal Society London, A382, 25-42, 1982.

2.4 Kolpakov, A.G., "On the determination of the averaged moduli of elastic gridworks", Prikl. Mat. Mekh, 59, 969-977, 1985.

2.5 Almgren, R. F., "An isotropic three dimensional structure with Poisson's ratio $=-1$ ", $J$. Elasticity, 15, 427-430, 1985.

2.6 Warren, T. L., "Negative Poisson's ratio in a transversely isotropic foam structure", J. Appl. Physics, 67, 7591-7594 1990.

2.7 Gibson, L. J., Ashby, M. F.,Cellular solids, Pergamon, 1988.

2.8 Lakes, R. S., "Foam structures with a negative Poisson's ratio", Science , 235, 1038-1040, 1987.

2.9 Friis, E.A., Lakes, R. S., and Park, J.B., "Negative Poisson's ratio polymeric and metallic materials", Journal of Materials Science, 23, 4406-4414, 1988.

2.10 Choi, J. B. and Lakes, R. S., "Nonlinear properties of metallic cellular materials with a negative Poisson's ratio", J. Materials Science, 27, 5373-5381, 1992.

2.11 Choi, J. B. and Lakes, R. S., "Nonlinear properties of polymer cellular materials with a negative Poisson's ratio", J. Materials Science, 27, 4678-4684, 1992.

2.12 Caddock, B.D. and Evans, K.E., "Microporous materials with negative Poisson's ratio: I. Microstructure and mechanical properties", J. Phys. D., Appl. Phys., 22, 1877-1882, 1989.

2.13 Evans, K.E. and Caddock, B.D., "Microporous materials with negative Poisson's ratio: II. Mechanisms and interpretation", J. Phys. D., Appl. Phys., 22, 1883-1887, 1989.

2.14 Herakovich, C.T., "Composite laminates with negative through-the -thickness Poisson's ratio", J. Composite Materials , 18, 447-455, 1985.

2.15 Tsai, S.W. and Hahn, H.T., Introduction to composite materials, Technomic, Lancaster PA 1980.

2.16 Miki, M. and Morotsu, Y. "The peculiar behavior of the Poisson's ratio of laminated fibrous composites", JSME International Journal, 32, 67-72, 1989.

2.17 Love, A.E.H., A Treatise on the Mathematical Theory of Elasticity, 4th ed, Dover, NY, 1944.

2.18 Simmons, G. and Wang, H., Single crystal elastic constants and calculated aggregate properties: a handbook, MIT Press, Cambridge, 2nd ed, 1971.

2.19 Milton, G., "Composite materials with Poisson's ratios close to -1", J. Mech. Phys. Solids, 40, 1105-1137, 1992.

2.20 Weiner, J.H., Statistical Mechanics of Elasticity, J. Wiley, NY, 1983.

2.21 Timoshenko, S. P., History of Strength of Materials, Dover, NY, 1983.

2.22 Lakes, R. S., "Negative Poisson's ratio materials", Science, 238, 551, 1987.

2.23 Burns, S., "Negative Poisson's ratio materials", Science, 238, 551, 1987.

2.24 Lakes, R. S., "Deformation mechanisms of negative Poisson's ratio materials: structural aspects", J. Materials Science, 26, 2287-2292, 1991.

2.25 Wojciechowski, K.W., "Two-dimensional isotropic system with a negative Poisson ratio", Physics Letters A, 137, 60-64, 1989.

2.26 Bathurst, R.J. and Rothenburg, L., "Note on a random isotropic granular material with negative Poisson's ratio", Int. J. Engng. Sci, 26, 373-383, 1988.

2.27 Rothenburg, L., Berlin, A. A. and Bathurst, R.J., "Microstructure of isotropic materials with negative Poisson's ratio", Nature, 354, 470-472, 1991.

2.28 Chen, C.P. and Lakes, R. S., "Holographic study of conventional and negative Poisson's ratio metallic foams: elasticity, yield, and micro-deformation", J. Materials Science, 26, 5397-5402, 1991.

2.29 Evans, K.E., Nkansah, M. A., Hutchinson, I. J., and Rogers, S. C., "Molecular network design", Nature, 353, 124, 1991. 
2.30 Lakes, R. S., "Advances in negative Poisson's ratio materials", Advanced Materials (Weinheim, Germany), 5, 293-296, 1993.

2.31 Chen, C.P. and Lakes, R. S., "Dynamic wave dispersion and loss properties of conventional and negative Poisson's ratio polymeric cellular materials", Cellular Polymers, 8, 343-359, 1989.

2.32 Choi, J. B. and Lakes, R. S., "Design of a fastener based on negative Poisson's ratio foam", Cellular Polymers, 10, 205-212, 1991.

3.1 Chen, C. P., and Lakes, R. S. "Viscoelastic behaviour of composite materials with conventional- or negative-Poisson's-ratio foam as one phase," Journal of Materials Science, 28, 4288-4298, 1993.

3.2 Lee, E. H. "Stress analysis in viscoelastic bodies", Q. Appl. Math, 13: 183-190, 1955.

3.3 Christensen, R. M.Theory of Viscoelasticity, Academic, NY, second edition, 1982.

3.4 Brodt, M. and Lakes, R. S., "Composite materials which exhibit high stiffness and high viscoelastic damping", J. Composite Materials, 29, 1823-1833, 1995.

3.5 Gibiansky, L. V. and Lakes, R. S. "Bounds on the complex bulk modulus of a two-phase viscoelastic composite with arbitrary volume fractions of the components", Mechanics of Materials, 16: 317-331, 1993.

3.6 Brodt, M., Cook, L. S., and Lakes, R. S., "Apparatus for measuring viscoelastic properties over ten decades: refinements", Review of Scientific Instruments, 66(11), in press, Nov. 1995.

4.1 Timoshenko, S.P., History of Strength of Materials, Dover, 1983.

4.2 Sokolnikoff, I.S., Mathematical Theory of Elasticity, Krieger, 1983.

4.3 Fung, Y.C., Foundations of Solid Mechanics, Prentice Hall, 1968.

4.4 Cosserat, E. and Cosserat, F., Theorie des Corps Deformables, Hermann et Fils, Paris, 1909.

4.5 Voigt, W., "Theoretische Studien uber die Elasticitatsverhaltnisse der Krystalle", Abh. Ges. Wiss. Gottingen, 34, 1887.

4.6 Aero, E. L. and Kuvshinskii, E. V., "Fundamental equations of the theory of elastic media with rotationally interacting particles", Fizika Tverdogo Tela, 2, 1399-1409, 1960; Translation, Soviet Physics-Solid State 2, 1272-1281, 1961.

4.7 Mindlin, R. D., "Stress functions for a Cosserat continuum", Int. J. Solids and Structures 1, 265-271, 1965.

4.8 Mindlin, R. D. and Tiersten, H. F., "Effect of couple stresses in linear elasticity", Arch. Rational Mech. Analy, 11, 415-448, 1962.

4.9 Eringen, A.C. Theory of micropolar elasticity. In Fracture, Vol. 1, 621-729 (edited by H. Liebowitz), Academic Press, 1968.

4.10 Nowacki, W., Theory of micropolar elasticity, Poznan, 1970.

4.11 Cowin, S. C., "Stress functions for Cosserat elasticity", Int. J. Solids, Structures, 6, 389-398, 1970.

4.12 Gauthier, R. D. and W. E. Jahsman. "A quest for micropolar elastic constants." J. Applied Mechanics, 42, 369-374, 1975.

4.13 Cowin, S. C. "An incorrect inequality in micropolar elasticity theory." J. Appl. Math. Phys. (ZAMP) 21, 494-497, 1970 b.

4.14 Lakes, R.S., "A pathological situation in micropolar elasticity, J. Applied Mechanics, 52 2342351985.

4.15 Krishna Reddy, G. V. and Venkatasubramanian, N. K., "On the flexural rigidity of a micropolar elastic circular cylinder", J. Applied Mechanics, 45, 429-431, 1978.

4.16 Mindlin, R. D., "Effect of couple stresses on stress concentrations", ExperimentalMechanics, 3, 1-7, 1963.

4.17 Lakes, R. S. and Benedict, R. L., "Noncentrosymmetry in micropolar elasticity." International Journal of Engineering Science, 29, 1161-1167, 1982.

4.18 Lakhtakia, A., Varadan, V. K., and Varadan, V. V., "Elastic wave propagation in noncentrosymmetric, isotropic media: dispersion and field equations", J. Applied Physics, 64, 5246, 1988.

4.19 Ellis, R.W. and Smith, C.W., "A thin plate analysis and experimental evaluation of couple stress effects", Experimental Mechanics, 7, 372-380, 1968. 
4.20 Yang, J. F. C. and Lakes, R. S., "Transient study of couple stress effects in human compact bone", J. Biomechanical Engineering, 103, 275-279, 1981.

4.21 Yang, J. F. C. and R. S. Lakes. "Experimental study of micropolar and couple-stress elasticity in bone in bending." J. Biomechanics, 15, 91-98, 1982.

4.22 Lakes, R.S. and Yang, J.F.C., "Micropolar elasticity in bone: rotation modulus $\kappa "$ ", Proceedings 18th Midwest Mechanics Conference, Iowa City, Developments in Mechanics, Vol. 12, p. 239-242, 1983.

4.23 Ascenzi, A., Baschieri, P., and Benvenuti, A., "The torsional properties of selected single osteons", J. Biomechanics, 27, 875-884, 1994.

4.24 Lakes, R. S., "On the torsional properties of single osteons", J. Biomechanics, 28, 1409-1410, 1995.

4.25 Lakes, R. S., "Experimental microelasticity of two porous solids", Int. J. Solids, Structures, 22, 55-63, 1986.

4.26 Anderson, W. B. and Lakes, R. S., "Size effects due to Cosserat elasticity and surface damage in closed-cell polymethacrylimide foam", Journal of Materials Science, 29, 6413-6419, 1994.

4.27 Park, H. C. and R. S. Lakes, "Torsion of a micropolar elastic prism of square cross section." Int. J. Solids, Structures, 23, 485-503, 1987.

4.28 Park, H. C. and R. S. Lakes. "Cosserat micromechanics of human bone: strain redistribution by a hydration-sensitive constituent." J. Biomechanics, 19, 385-397, 1986.

4.29 Lakes, R. S., Gorman, D. and Bonfield, W., "Holographic screening method for microelastic solids." J. Materials Science, 20, 2882-2888, 1985.

4.30 Lakes, R. S. "Demonstration of consequences of the continuum hypothesis." Mechanics Monograph, M5, 1-5, 1985.

4.31 Lakes, R. S. and Yang, J. F. C., "Concentration of strain in bone", Proceedings 18th Midwest Mechanics Conference, Developments in Mechanics 12, 233-237, 1983.

4.32 Hlavacek, M., "A continuum theory for fibre reinforced composites", Int. J. Solids and Structures, 11, 199-211, 1975.

4.33 Hlavacek, M., "On the effective moduli of elastic composite materials", Int. J. Solids and Structures, 12, 655-670, 1976.

4.34 Herrmann, G. and Achenbach, J. D., "Applications of theories of generalized continua to the dynamics of composite materials", in Mechanics of Generalized Continua, ed. E. Kröner, Springer Verlag, 1967.

4.35 Berglund, K., "Structural models of micropolar media", in Mechanics of Micropolar Media, edited by O. Brulin and R. K. T. Hsieh, World Scientific, Singapore, 1982.

4.32 Lakes, R. S., "Experimental methods for study of Cosserat elastic solids and other generalized continua", in Continuum models for materials with micro-structure, ed. H. Mühlhaus, J. Wiley, Ch. 1, p. 1-22, 1995.

5.1 Lakes, R. S., "Materials with structural hierarchy", Nature, 361, 511-515, 1993.

5.2 Loyrette, H. Gustave Eiffel, Rizolli, NY, 1985.

5.3 Gibson, L. J. and Ashby, M. F., Cellular solids, Pergamon, Oxford, 1988.

5.4 Katz, J. L., "Hard tissue as a composite material- I. Bounds on the elastic behavior", $J$. Biomechanics 4, 455-473, 1971.

5.5 Piekarski, K., "Fracture of bone", J. Appl. Phys. 41, 215-223, 1970.

5.6 Lakes, R.S. and Saha, S., "Cement line motion in bone," Science, 204, 501-503, 1979.

5.7 Currey, J., The mechanical adaptations of bones, Princeton University Press, 1984.

5.8 Piekarski, K. and Munro, M., "Transport mechanism operating between blood supply and osteocytes in long bones", Nature, 269, 80-82, 1977.

5.9 Katz, J. L., "Anisotropy of Young's modulus of bone", Nature, 283, 106-107 1980. 


\section{Figures}

\section{One length scale}

\section{Length scales}

Poisson's ratio $=1 / 4$, brittle

Two length scales

Poisson's ratio form -1 to $1 / 2$

Foam rib length and thickness

control Poisson's ratio by cell shape

Composite materials

Viscoelastic composites

Many length scales

Biological materials: bone, ligament

Hierarchical cellular solids: high strength

Hierarchical composites, laminates

The largest length scale

Cosserat elasticity

Toughness

1.1 Phenomena associated with length scales in the structural view, linked to continuum concepts.

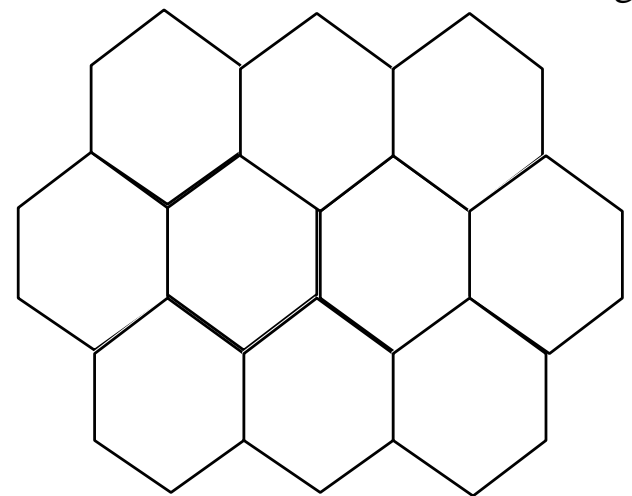

a

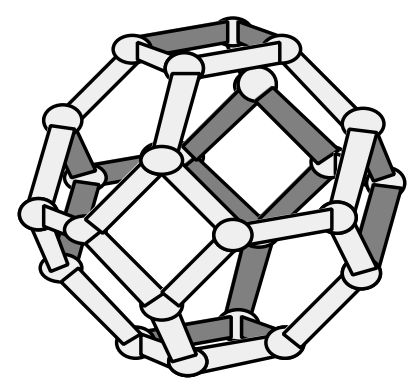

c

2.1 Honeycomb with positive (a) and negative (b) Poisson's ratio [redrawn after 2.3,2.4].

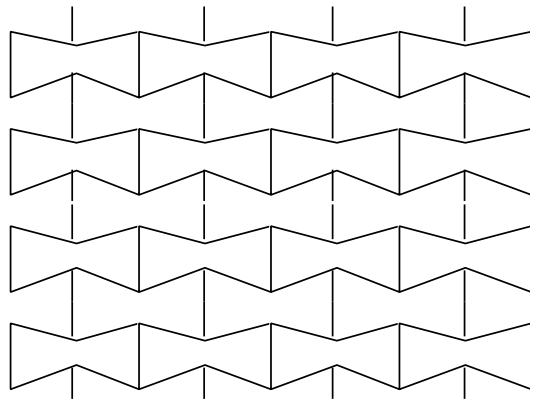

b

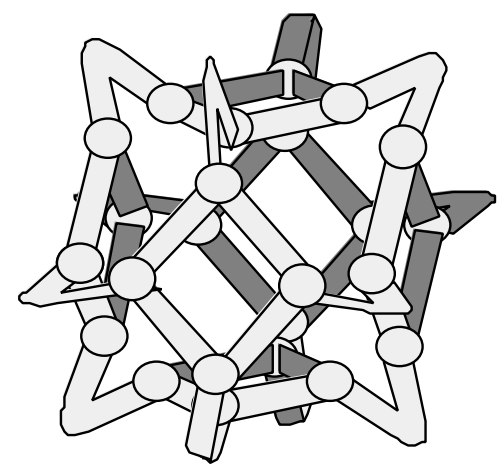

d 


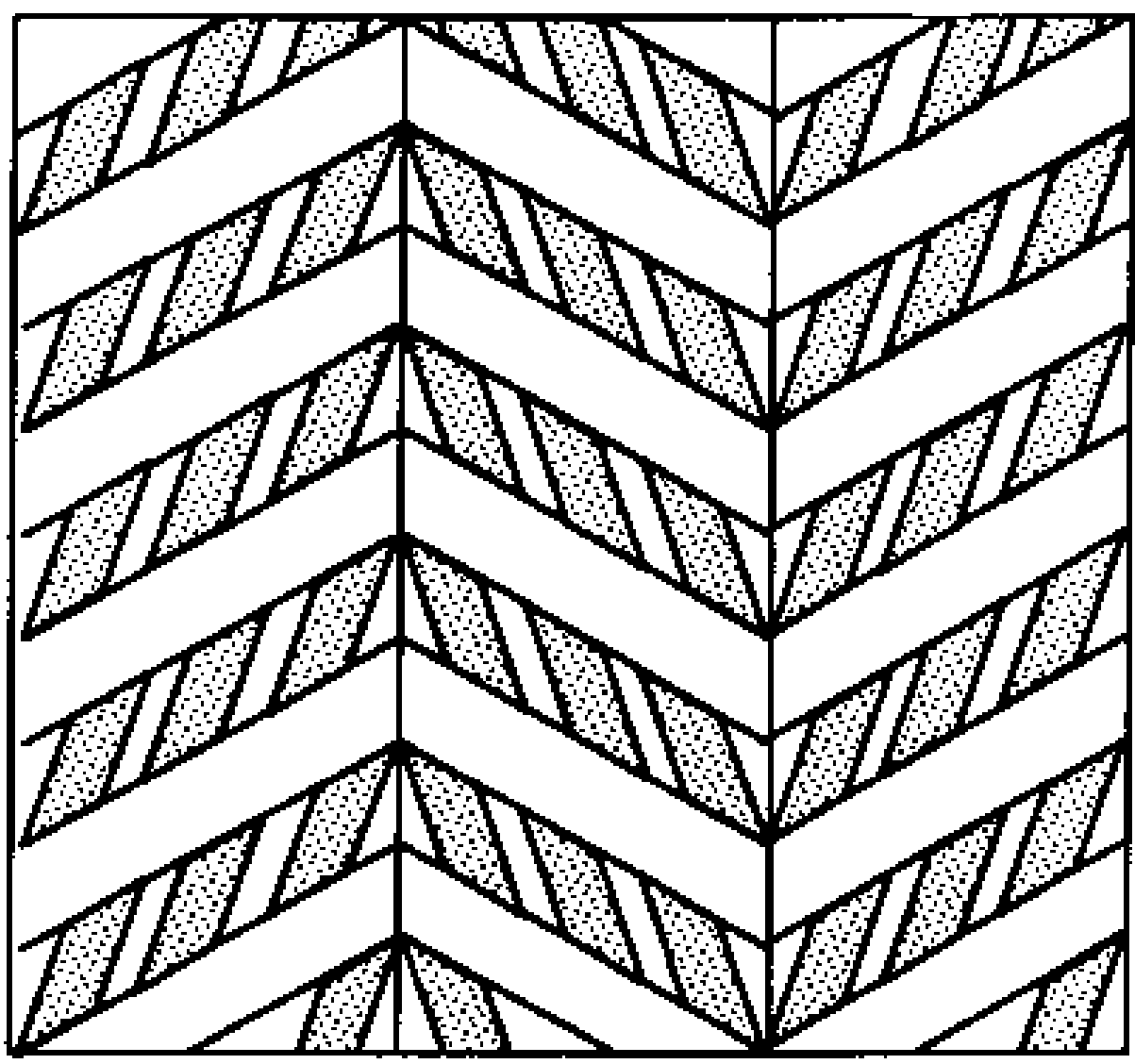

2.2 Laminate with negative Poisson's ratio, redrawn after Milton [2.19].

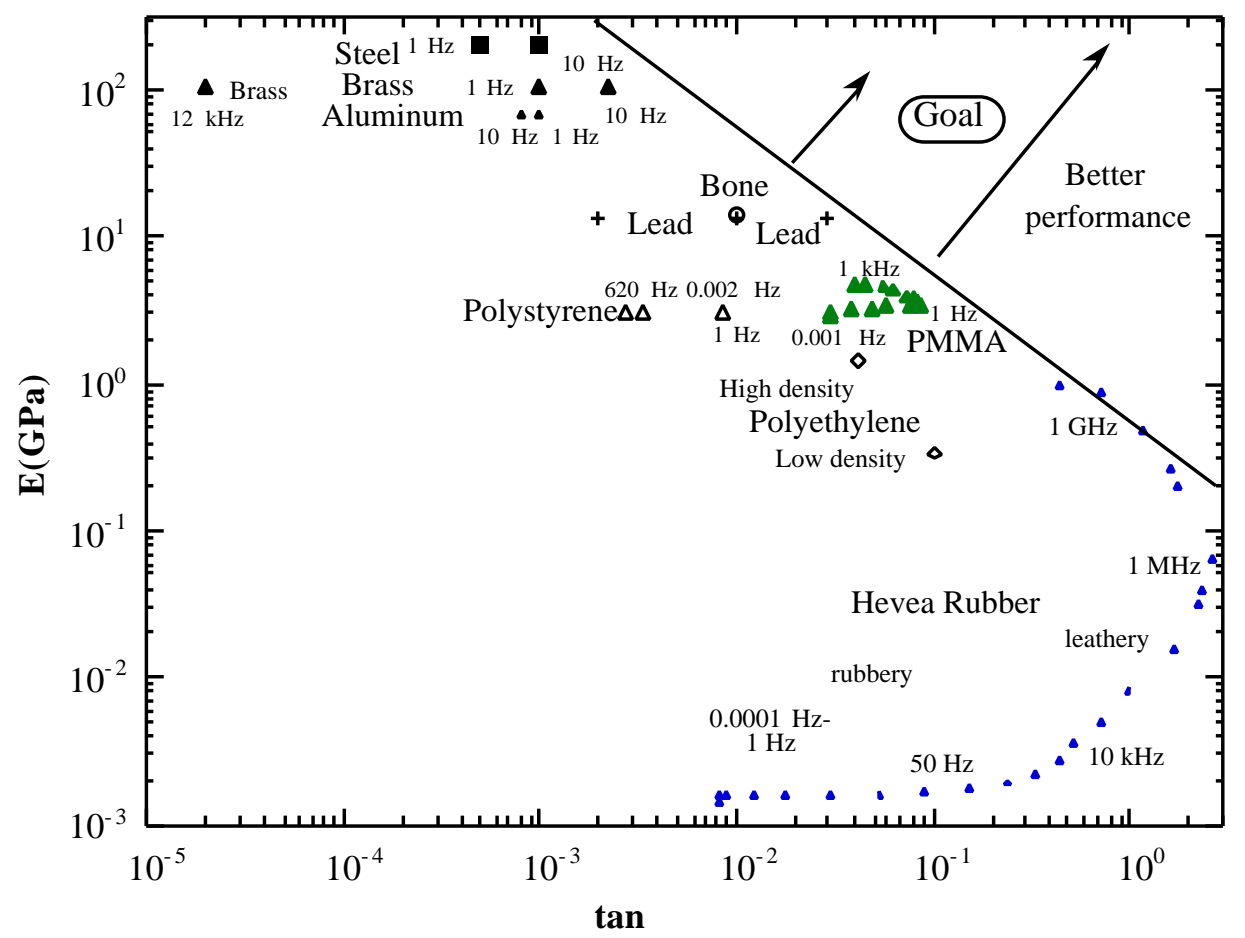

3.1 Stiffness-loss map showing typical materials. 


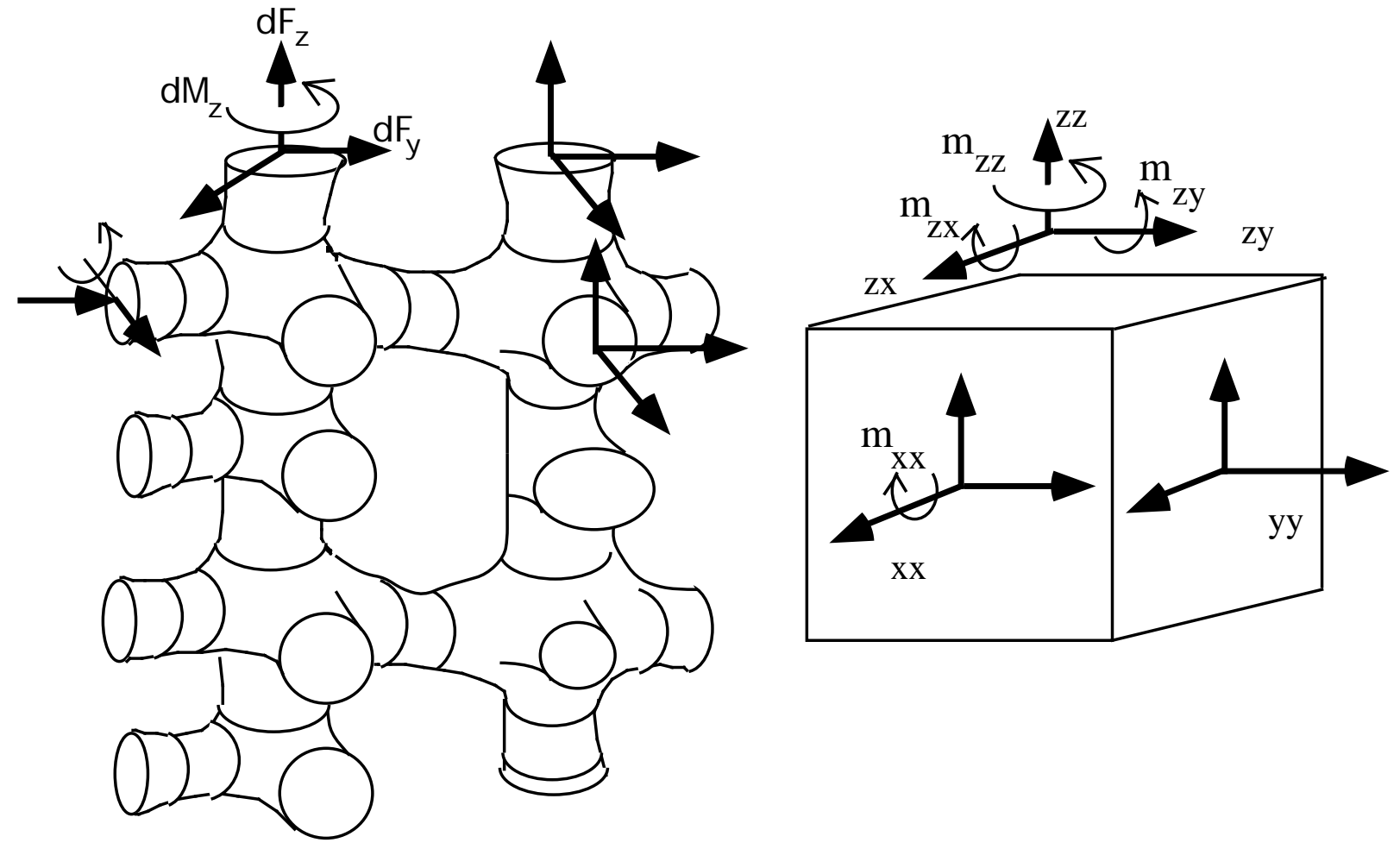

4.1 Components of stress and couple stress.

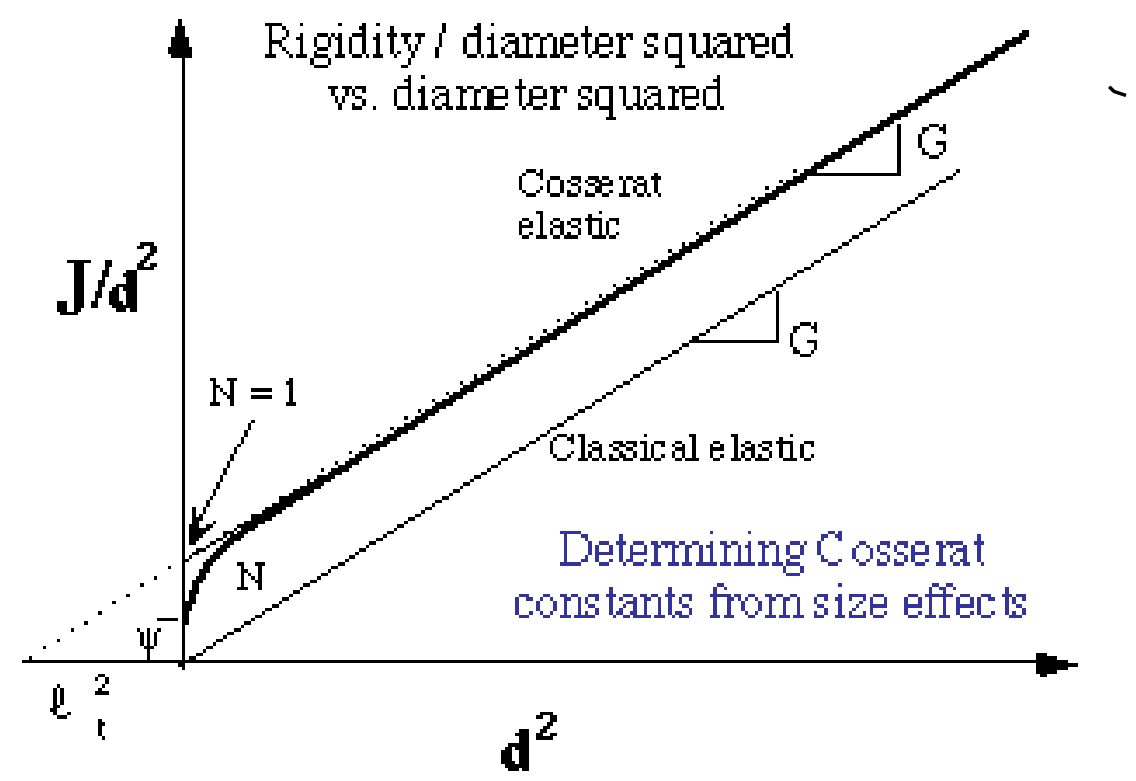

4.2 Size effects in torsion of a circular cylindrical rod. Rigidity/ diameter squared vs diameter squared, for a classical solid and a Cosserat solid. 


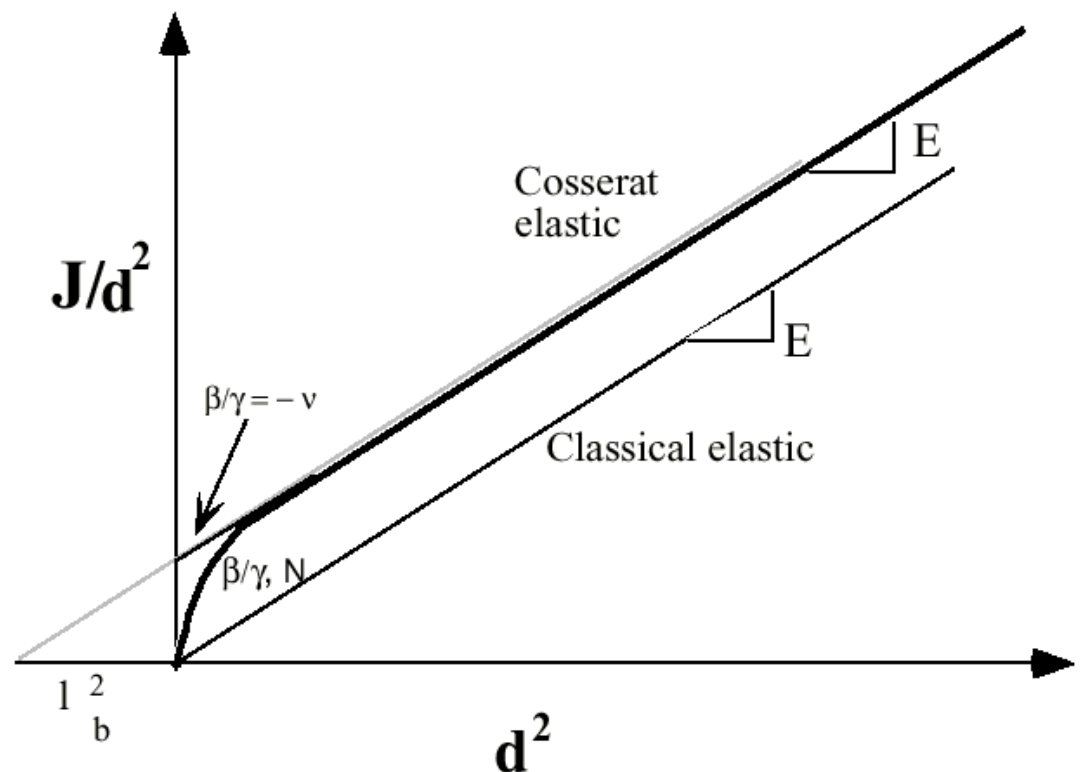

4.3 Size effects in bending of a circular cylindrical rod. Rigidity/ diameter squared vs diameter squared, for a classical solid and a Cosserat solid. 


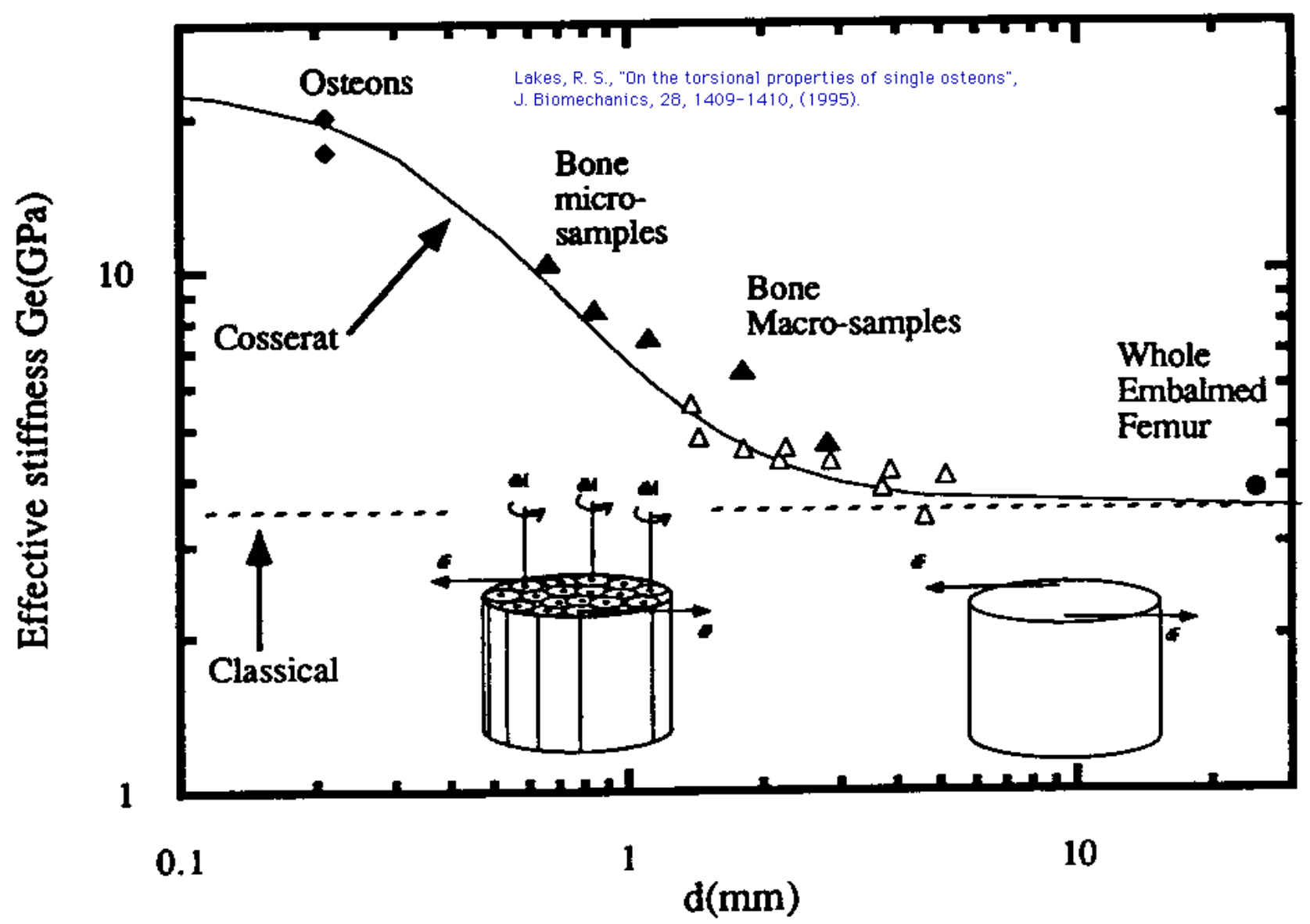

4.4 Effective torsional stiffness vs diameter for bone, adapted from [4.24].

- Osteons (large fibers in bone), fresh, wet, after Ascenzi, et. al., [4.23].

$\Delta \quad$ Bone macro-samples, fresh, wet, after Yang and Lakes [4.20].

$\Delta \quad$ Bone micro-samples, fresh, wet, after Lakes and Yang [4.23].

- Whole embalmed femur, after Huiskes, et. al., (adduced in [4.24]).

Solid curve, Cosserat elasticity, torsion, assuming technical elastic constants to be:

$\mathrm{G}=3.5 \mathrm{GPa}, \mathrm{I}_{\mathrm{t}}=0.22 \mathrm{~mm}, \mathrm{I}_{\mathrm{b}}=0.44 \mathrm{~mm}, \Psi=1.5, \mathrm{~N}=0.62$.

Apparent shear modulus seems to vary with diameter.

Inset drawings: right, classical elastic continuum with stress due to force increments dF;

left, bone as a Cosserat elastic continuum with both force increments $\mathrm{dF}$ and torque increments $\mathrm{dM}$ upon osteons.

- - - Dashed line: classical elasticity, torsion. Classical apparent shear modulus is independent of diameter. 


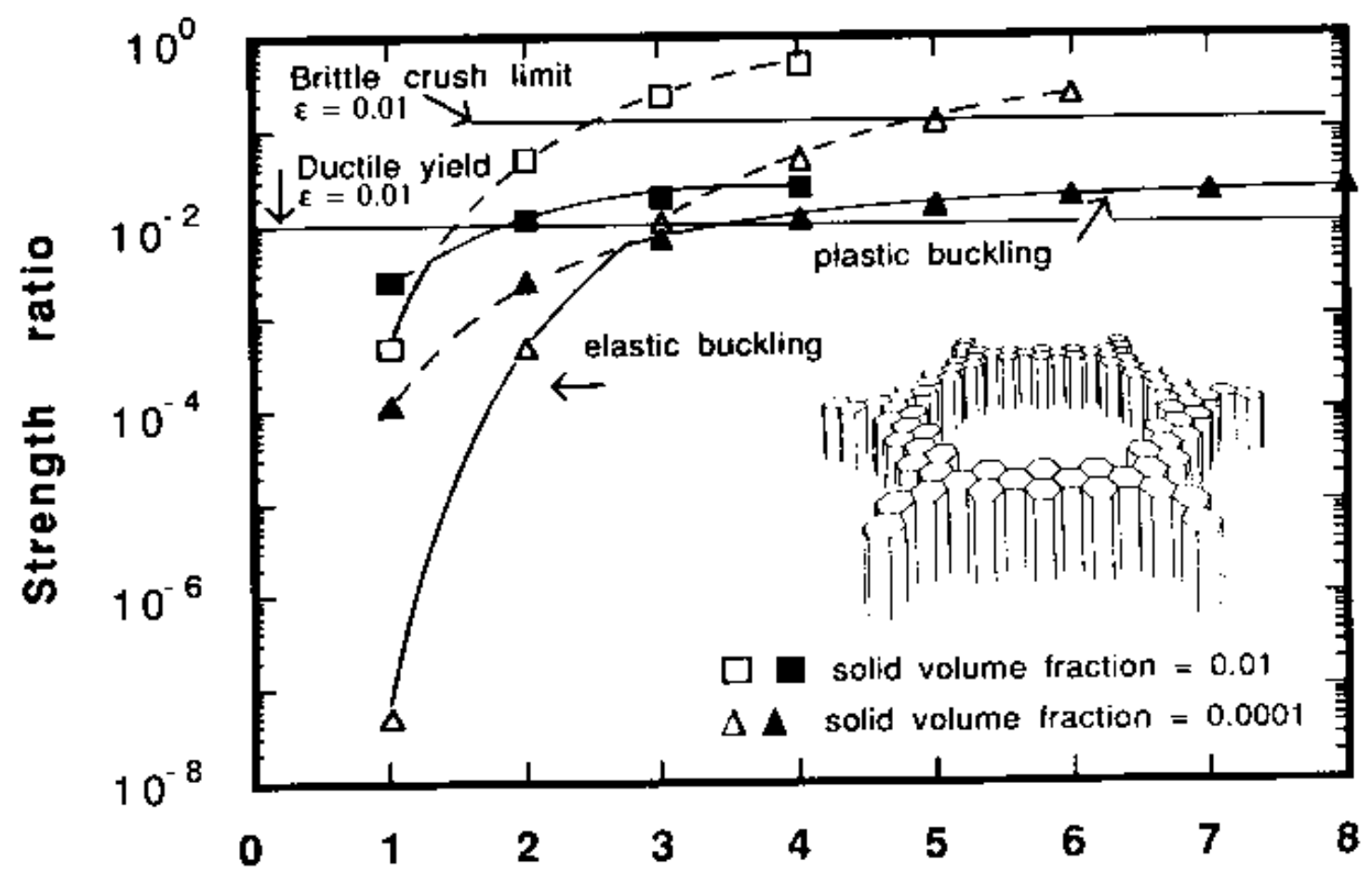

Lakes, R. S., Hierarchical order $n$

"Materials with structural hierarchy",

Nature. 361. 511-515 (1993).

5.1 Strength to density ratio of hierarchical honeycomb increases with hierarchical order $\mathrm{n}$. Normalized strength ratio $\left(\sigma_{n} / \rho_{n}\right) /\left(E_{o} / \rho_{o}\right)$, for hierarchical honeycomb microstructure, as a function of hierarchical order $n$, for several solid volume fractions $\rho_{n} / \rho_{o}$ : squares, 0.01 ; triangles 0.0001. For the solid the yield strain and fracture strain are assumed to be 0.01 . Open symbols: elastic buckling. Solid symbols: plastic buckling. Inset: second order honeycomb cell. Solid curves: buckling mode with lowest stress limits the strength. Adapted from $\{5.1]$. 